\title{
General practitioner management of genetic aspects of a cardiac disease: a scenario-based study to anticipate providers' practices
}

\author{
Kirsty Challen • Hilary Harris • Ulf Kristoffersson • Irmgard Nippert • \\ Joerg Schmidtke • Leo P. Ten Kate • Caroline Benjamin • Elizabeth Anionwu • \\ Anne-Marie Plass • Claire Julian-Reynier • Rodney Harris
}

Received: 3 February 2010 / Accepted: 28 July 2010 / Published online: 9 August 2010

(C) The Author(s) 2010. This article is published with open access at Springerlink.com

\begin{abstract}
It is increasingly recognised that genetics will have to be integrated into all parts of primary health care. Previous research has demonstrated that involvement and confidence in genetics varies amongst primary care providers. We aimed to analyse perceptions of primary care providers regarding responsibility for genetic tasks and factors affecting those perceptions. Postal questionnaire including a hypothetical case management scenario of a cardiac condition with a genetic component was sent to random samples of medically qualified general practitioners in France, Germany, Netherlands, Sweden and UK $(n=1,168)$. Logistic regres-
\end{abstract}

K. Challen $(\bowtie) \cdot H$. Harris $\cdot$ R. Harris

GenEd Co-ordinating Centre, University of Manchester,

Manchester, UK

e-mail: kirstychallen@hotmail.com

H. Harris

e-mail: hilaryharris@btinternet.com

R. Harris

e-mail: rharrisgened@btinternet.com

U. Kristoffersson

Department of Clinical Genetics, University and Regional

Laboratories, Lund University,

Lund, Sweden

e-mail: Ulf.kristoffersson@med.lu.se

\section{Nippert}

Women's Health Research, Westfaelische Wilhelms-Universitaet

Muenster,

Muenster, Germany

e-mail: nippert@uni-muenster.de

J. Schmidtke

Insititut fur Humangenetik, Medizinische Hochschule Hannover, Hannover, Germany

e-mail: Schmidtke.joerg@mh-hannover.de sion analysis of factors affecting primary care practitioners' willingness to carry out genetic tasks themselves was conducted; $61 \%$ would take a family history themselves but only $38 \%$ would explain an inheritance pattern and $16 \%$ would order a genetic test. In multivariate analysis, only the country of practice was consistently predictive of willingness to carry out genetic tasks, although male gender predicted willingness to carry out the majority of tasks studied. The stage of career at which education in genetics had been provided was not predictive of willingness to carry out any of the tasks analysed. Country of practice is significantly predictive of attitudes

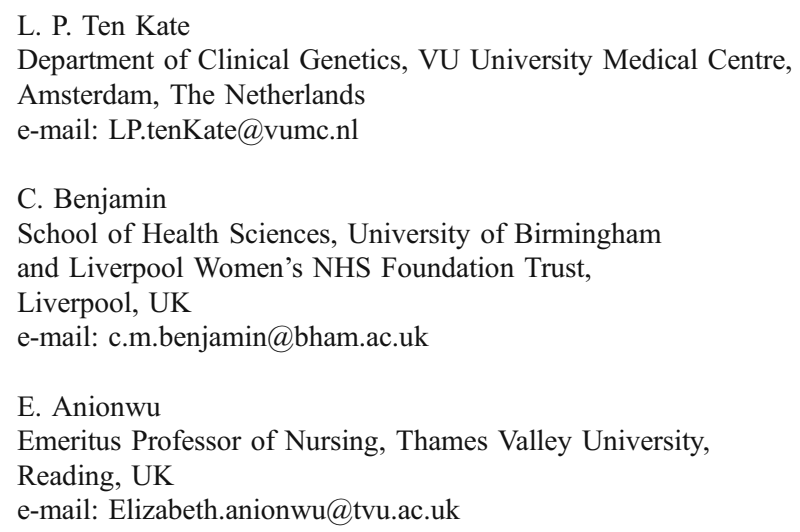


towards genetics in primary care practice and therefore genetic education structure and content in Europe will need to be significantly tailored towards country-specific approaches.

Keywords Genetics · Primary care $\cdot$ Education

\section{Background}

Over the last decade, basic scientific research has led to a greater understanding of the contribution made by genes to present and future health (Guttmacher and Collins 2002). It is increasingly recognised that genetic information will need to be integrated into all aspects of health care delivery, including primary care (Department of Health 2003; Greendale and Pyeritz 2001; Harris and Harris 1995). Patient advocacy groups have lobbied to raise health professionals' awareness of genetic issues (World Alliance of Organizations for the Prevention of Birth Defects 2004), and the need for both patients and professionals to have an appropriate level of familiarity with the new technologies has been recognised by the European Commission (McNally et al. 2004).

Primary care providers have varying levels of involvement and confidence in genetics (Emery et al. 1999). We have demonstrated variable quality care provided for genetic conditions by non-geneticists (Harris et al. 1999). This has also been reported in Australia (Tyzack and Wallace 2003), the Netherlands (Baars et al. 2003; van Langen et al. 2003), Singapore (Yong et al. 2003), and USA (Barrison et al. 2003; Batra et al. 2002; Schroy et al. 2002; Taylor 2003). Core competencies for all health professionals and particular professional groups are being developed by expert panels (Calzone et al. 2002; Core Competency Working Group of the National Coalition for Health Professional Education in Genetics 2001; Kirk et al. 2003), and we have recently reported the educational priorities of the healthcare providers themselves (JulianReynier et al. 2008). A multiplicity of structures and organisations are involved in training health professionals in genetics (Challen et al. 2006, 2005; Henriksson and Kristoffersson 2006; Julian-Reynier and Arnaud 2006; Plass et al. 2006; Schmidtke et al. 2006). As part of a larger study in five European countries, we examined the self-reported behaviours and educational priorities of primary care providers in situations where genetics was relevant. This paper will present the results relating to perceptions of professional responsibility for genetic care amongst general practitioners, using hereditary cardiac disease as an example of the "new" genetics in common diseases. We aimed to analyse these attitudes and their determining factors.

\section{Methods}

\section{Sampling}

As part of the larger GenEd (Genetic Education for Nongenetic Health Professionals) study into educational priorities in genetics for primary care providers, general practitioners in France, Germany, Netherlands, Sweden and UK were sent a self-administered questionnaire in early 2005. The sample size was calculated based on a $10 \%$ precision $(95 \% \mathrm{CI})$ for an educational outcome measure (Calefato et al. 2008). Germany used a deliberate oversampling strategy because of the anticipated low response rate. In France and UK, a random sample of a representative database was taken, in Germany a random sample of MDs receiving reimbursement from sickness funds and training MD students was taken, in the Netherlands sampling was undertaken by the Netherlands Institute for Health Services Research excluding those who had recently participated in research and Sweden all general practitioners were approached. Non-responders were sent at least one reminder letter and, in some countries, were telephoned.

\section{Questionnaire}

The questionnaire was developed by a multidisciplinary group including geneticists, primary care providers and statisticians, initially in English. It was piloted in English in each participating country, then translated and backtranslated to ensure consistency. Translated questionnaires were then re-piloted. As well as demographics, the questionnaire included a hypothetical scenario relating to sudden cardiac death, a diagnosis chosen because of the increasing recognition of genetic factors in its aetiology (as demonstrated by its inclusion in the 2005 revision of the UK National Service Framework for Heart Disease (Department of Health 2005)), but where "traditional" genetic teaching is unlikely to have featured. The text is shown in the text box. The vignette may have provided new information to some respondents. We wished to standardise their knowledge in order to interpret their subsequent practice intentions, as we intended the survey to be a pragmatic study of usual practice rather than a specific test of knowledge of HOCM.

Box: text of the questionnaire scenario

Mr Smith (aged 35) attends your surgery because his 27-year-old brother, a competitive swimmer, has just died suddenly. He collapsed in the pool and despite defibrillation was found to be dead. Although sudden death might not immediately suggest a genetic condition $\mathrm{Mr}$ Smith is worried because his mother's sister died suddenly aged 30 and he asks whether the same may happened to him, his children Melanie (12 years), and Tom (6 months) or his brother (32) or sister (24). 
He has been told that his brother's post-mortem demonstrated hypertrophic obstructive cardiomyopathy (HCM), which can be inherited as an autosomal dominant condition. $80 \%$ of non-traumatic sudden deaths in young athletes are due to inherited or congenital cardiovascular abnormalities and HCM accounts for $40-50 \%$ of these. Genetic testing may lead to identification of patients at high risk for sudden death as early as 10 years of age. Treatment can be considered with implantable defibrillators or medication.

Respondents were asked who, in the scenario, should perform the following tasks, with options being "myself without seeking further information", "myself after consulting a journal or the web", "myself after consulting a colleague", "a genetic specialist", "a cardiologist":

- Taking a family history

- Explaining the inheritance pattern

- Explaining the risk to the patient's children

- Giving information about available gene tests

- Informing the patient of the implications if no mutation were to be found

- Informing the patient of the implications if a mutation were to be found

- Ordering the genetic test

- Explaining the test result

- Explaining the implications of the test result for the patient's children

Statistical analysis

Responses were entered into an SPSS v11.0 data sheet using SNAP v7.0 questionnaire and scanning software. For each task addressed in the questionnaire, the five possible responses were dichotomised into "likely to do oneself" and "should be done by a different professional". Univariate analysis was carried out for all tasks for association with: country of practice, gender, age (over/under 50 years), years in practice (under 10,11-20, over 20), highest level of education in genetics, and usefulness or otherwise of continuing medical education, specialist training and undergraduate training. Factors found to be predictive at univariate analysis of "likely to do oneself" were entered into multivariate stepwise logistic regression analysis using a forward procedure (Wald test) (Hosmer and Lemeshow 2000). A type 1 error of $<0.05$ was chosen for the variables to be included in the final model.

Ethics

Ethical approval was provided by the Eastern MREC (UK) and appropriate approval was obtained in all countries.

\section{Results}

Overall, 1,168 (28.6\%) practitioners responded (France 236 (48.7\%), Germany 251 (20.8\%), Netherlands 254 (37\%), Sweden $262(38.7 \%)$, UK $165(23.1 \%))$. Demographics of respondents are shown in Table 1 . The highest level of genetic education varied significantly $(p<0.05)$ between

Table 1 Demographics of respondents ( $n$ varies due to incomplete responses)

\begin{tabular}{|c|c|}
\hline Characteristic & Number (percentage) \\
\hline \multicolumn{2}{|l|}{ Country of practice } \\
\hline France & $236(20.2)$ \\
\hline Germany & $251(21.5)$ \\
\hline Netherlands & $254(21.7)$ \\
\hline Sweden & $262(22.4)$ \\
\hline UK & $165(14.1)$ \\
\hline \multicolumn{2}{|l|}{ Gender } \\
\hline Male & $764(65.4)$ \\
\hline Female & $404(34.6)$ \\
\hline \multicolumn{2}{|l|}{ Age group } \\
\hline$\leq 50$ years & $572(49.0)$ \\
\hline$>50$ years & $596(51.0)$ \\
\hline \multicolumn{2}{|l|}{ Years in practice } \\
\hline$\leq 10$ & $182(15.6)$ \\
\hline $11-20$ & $466(39.9)$ \\
\hline$>20$ & $520(44.5)$ \\
\hline \multicolumn{2}{|l|}{ Patients seen per week } \\
\hline$<25$ & $33(2.9)$ \\
\hline $26-50$ & $133(11.5)$ \\
\hline $51-100$ & $358(31.0)$ \\
\hline $101-150$ & $309(26.8)$ \\
\hline $151-200$ & $199(17.2)$ \\
\hline$>200$ & $122(10.6)$ \\
\hline \multicolumn{2}{|c|}{ Highest level of education in genetics } \\
\hline None & $224(19.2)$ \\
\hline Undergraduate & $680(58.2)$ \\
\hline During specialist training & $53(4.5)$ \\
\hline CME & $172(14.7)$ \\
\hline Further degree & $32(2.7)$ \\
\hline Missing & $7(0.6)$ \\
\hline \multicolumn{2}{|c|}{ Value of undergraduate training $(n=880)$} \\
\hline Useful & $538(61.1)$ \\
\hline Useless & $342(38.9)$ \\
\hline \multicolumn{2}{|c|}{ Value of specialist training $(n=71)$} \\
\hline Useful & $61(85.9)$ \\
\hline Useless & $10(14.1)$ \\
\hline \multicolumn{2}{|l|}{ Value of CME $(n=172)$} \\
\hline Useful & $164(95.3)$ \\
\hline Useless & $8(4.7)$ \\
\hline
\end{tabular}


Table 2 Highest level of education by years in practice

\begin{tabular}{lcccccc}
\hline & Undergraduate & Specialist & CME & Degree & None & Total \\
\hline$\leq 10$ years & 130 & 16 & 19 & 4 & 12 & 181 \\
$11-20$ years & 309 & 18 & 60 & 10 & 65 & 462 \\
$>20$ years & 241 & 19 & 93 & 18 & 147 & 518 \\
Total & 680 & 53 & 172 & 32 & 224 & 1161 \\
\hline
\end{tabular}

countries; rates of receiving relevant undergraduate education were: Sweden $90 \%$, UK $65 \%$, Germany $60 \%$, Netherlands $57 \%$ and France $50 \%$. The highest level of genetic education was also significantly associated with years spent in practice (Table 2; $\chi^{2}=84.578, \mathrm{df}=8, p<0.001$ ).

Numbers of respondents willing to carry out each of the tasks themselves is shown in Table 3. Most (61\%) expected to take a family history, and a significant minority (38\%) were willing to explain an inheritance pattern. However, only $10.3(28 \%)$ were willing to carry out any other tasks. Univariate analysis of factors predicting likelihood of carrying out tasks oneself is shown in Table 4. Factors which remained significant at multivariate analysis are shown in Table 5. Only country of practice and gender were consistently predictive of willingness to carry out more complex tasks, with French/German and male GPs showing more willingness.

\section{Discussion}

Although most GPs (over 60\%) would consider it part of their role to take a family history, far fewer (less than 25\%) would be willing to discuss specific genetic tests or their implications. Taking a family history is generally considered essential for the appropriate management of genetic disorders. Thirty-eight per cent of GPs in this study felt that

Table 3 Willingness to carry out tasks oneself

\begin{tabular}{lll}
\hline Task & $\begin{array}{c}\text { Number willing } \\
\text { to perform task }\end{array}$ & Percentage \\
\hline Taking a family history & 717 & 61.4 \\
$\begin{array}{l}\text { Explaining the inheritance pattern } \\
\text { Explaining the genetic risk to }\end{array}$ & 445 & 38.1 \\
$\quad$ Mr Smith's children & 327 & 28 \\
$\begin{array}{l}\text { Giving information about available } \\
\text { genetic tests }\end{array}$ & 258 & 22.1 \\
$\begin{array}{l}\text { Informing Mr Smith of the implications } \\
\text { of no mutation being found }\end{array}$ & 316 & 27.1 \\
$\begin{array}{l}\text { Informing Mr Smith of the implications } \\
\text { of a mutation being found }\end{array}$ & 169 & 14.5 \\
$\begin{array}{l}\text { Ordering the genetic test } \\
\text { Explaining the test results }\end{array}$ & 183 & 15.7 \\
$\begin{array}{l}\text { Explaining the implications of the test } \\
\text { results for Mr Smith's children }\end{array}$ & 129 & 11 \\
\hline
\end{tabular}

this should be carried out by a specialist (either a geneticist or a cardiologist).

The country of practice was the only consistent predictor of GPs carrying out tasks themselves (with or without reference to a textbook, the web or a colleague), with French and German practitioners being more likely to do so. There appear to be two different patterns: German, Swedish and UK GPs were more likely to undertake initial tasks (particularly taking a family history), with lessening likelihood as the tasks became more complex, while French and Dutch GPs tended either to carry out a significant number of tasks or complete none and refer for the entire genetic care "package". It is unclear whether this reflects varying awareness or availability of specialist genetic services or varying willingness to refer to those services. It is likely that the health service model in each country will affect practitioners' expectations of managing the patient themselves or performing a gatekeeper role for secondary care. It may also be that varying health service structures restrict the availability of specific tests to non-specialist practitioners.

At least $50 \%$ of GPs recalled receiving undergraduate genetic education but this varied between countries. However, less than $10 \%$ recalled receiving genetic education during specialist training or continuing medical education, suggesting that any formal genetic education they had received was unlikely to have been up-to-date or clinically relevant. We could hypothesise that the counterintuitive finding (see Table 2) of those practitioners who had been practising longer having received more postspecialist training in genetics represents a "catch-up" phenomenon; those practitioners trained more recently received the same information during undergraduate or specialist training. The perceived usefulness of genetic education as an undergraduate was a positive predictor of likelihood to explain inheritance patterns, risks and gene tests. This may reflect increased comfort in discussing genetic issues amongst those practitioners who underwent early engagement with genetics.

Being male appeared to increase the likelihood of carrying out many genetic tasks, particularly the more complex ones. There are several possible contributors to this finding. The tasks we assessed were primarily biomedical, and significant literature demonstrates the tendency of male physicians to communicate biomedical 
Table 4 Univariate analysis

\begin{tabular}{|c|c|c|}
\hline Task & Variable & $\begin{array}{l}\text { Odds ratio for doing } \\
\text { oneself }(95 \% \mathrm{CI})\end{array}$ \\
\hline
\end{tabular}

\begin{tabular}{|c|c|c|}
\hline \multirow[t]{16}{*}{ Taking a family history } & \multicolumn{2}{|c|}{ Country (reference UK) } \\
\hline & France & $0.59(0.39-0.90)$ \\
\hline & Germany & $2.07(1.33-3.23)$ \\
\hline & Netherlands & $0.20(0.13-0.30)$ \\
\hline & Sweden & $2.41(1.54-3.79)$ \\
\hline & \multicolumn{2}{|c|}{ Gender (reference male) } \\
\hline & Female & $1.25(0.98-1.61)$ \\
\hline & \multicolumn{2}{|c|}{ Age (reference >50) } \\
\hline & $\leq 50$ & $0.73(0.57-0.92)$ \\
\hline & \multicolumn{2}{|c|}{ Years in practice (reference $>20$ ) } \\
\hline & $11-20$ & $0.90(0.69-1.16)$ \\
\hline & $\leq 10$ & $0.93(0.66-1.32)$ \\
\hline & \multicolumn{2}{|c|}{$\begin{array}{l}\text { Highest genetic education (reference } \\
\text { none) }\end{array}$} \\
\hline & Undergraduate & $1.45(1.07-1.98)$ \\
\hline & $\begin{array}{l}\text { During } \\
\text { specialist } \\
\text { training }\end{array}$ & $1.67(0.88-3.18)$ \\
\hline & $\mathrm{CME}$ & $0.52(0.35-0.78)$ \\
\hline
\end{tabular}

Value of genetic education (reference useless)

$\begin{array}{ll}\begin{array}{l}\text { Useful } \\ \text { undergraduate }\end{array} & 0.96(0.72-1.27) \\ \begin{array}{l}\text { Useful } \\ \text { specialist }\end{array} & 0.41(0.08-2.12) \\ \text { Useful CME } & 0.23(0.05-1.18) \\ \text { Country (reference } & \text { UK) } \\ \text { France } & 1.91(1.26-2.89) \\ \text { Germany } & 1.31(0.87-1.98) \\ \text { Netherlands } & 0.91(0.59-1.38) \\ \text { Sweden } & 1.48(0.98-2.23)\end{array}$

Gender (reference male)

Female $\quad 1.05(0.82-1.35)$

Age (reference $>50$ )

$\leq 50$

$1.44(1.14-1.83)$

Years in practice (reference $>20$ )

$$
\begin{array}{ll}
11-20 & 1.40(1.08-1.81) \\
\leq 10 & 1.23(0.87-1.74)
\end{array}
$$

Highest genetic education (reference none)

$$
\begin{array}{ll}
\text { Undergraduate } & 1.48(1.07-2.04) \\
\text { During } & 1.96(1.07-3.61) \\
\text { specialist } & \\
\text { training } & \\
\text { CME } & 1.09(0.71-1.67)
\end{array}
$$

\begin{tabular}{|c|c|c|}
\hline Task & Variable & $\begin{array}{l}\text { Odds ratio for doing } \\
\text { oneself }(95 \% \mathrm{CI})\end{array}$ \\
\hline & \multicolumn{2}{|c|}{ Gender (reference male) } \\
\hline & Female & $0.64(0.48-0.84)$ \\
\hline & \multicolumn{2}{|c|}{ Age (reference $>50$ ) } \\
\hline & $\leq 50$ & $1.20(0.93-1.55)$ \\
\hline & \multicolumn{2}{|c|}{ Years in practice (reference $>20$ ) } \\
\hline & $11-20$ & $1.03(0.78-1.36)$ \\
\hline & $\leq 10$ & $0.89(0.61-1.31)$ \\
\hline & \multicolumn{2}{|c|}{$\begin{array}{l}\text { Highest genetic education (reference } \\
\text { none) }\end{array}$} \\
\hline & Undergraduate & $1.05(0.75-1.47)$ \\
\hline & $\begin{array}{l}\text { During } \\
\text { specialist } \\
\text { training }\end{array}$ & $1.49(0.79-2.81)$ \\
\hline & CME & $0.89(0.57-1.40)$ \\
\hline
\end{tabular}

Value of genetic education (reference useless)

$$
\begin{array}{lc}
\begin{array}{l}
\text { Useful } \\
\text { undergraduate }
\end{array} & 1.55(1.17-2.05) \\
\text { Useful } & \\
\quad \text { specialist } & 1.45(0.37-5.66) \\
\text { Useful CME } & 0.84(0.19-3.65)
\end{array}
$$

Explaining the risk to Mr Smith's children

Table 4 (continued)

Value of genetic education (reference useless)

$$
\begin{array}{ll}
\begin{array}{l}
\text { Useful } \\
\text { undergraduate }
\end{array} & 1.50(1.10-2.05) \\
\text { Useful } & 1.62(0.38-6.88) \\
\text { specialist } & \\
\text { training } & \\
\text { Useful CME } & 0.56(0.13-2.43) \\
\text { Country (reference } & \mathrm{UK}) \\
\text { France } & 2.17(1.30-3.63) \\
\text { Germany } & 1.84(1.10-3.07) \\
\text { Netherlands } & 1.27(0.75-2.16) \\
\text { Sweden } & 1.59(0.95-2.67)
\end{array}
$$

Giving information about available gene tests

(1)

Informing Mr Smith of the implications if no mutation were to be found
Gender (reference male)

Female $\quad 0.63(0.46-0.85)$

Age (reference $>50$ )

$$
\leq 50 \quad 0.69(0.52-0.91)
$$

Years in practice (reference $>20$ )

$$
\begin{array}{ll}
11-20 & 0.79(0.59-1.07) \\
\leq 10 & 0.56(0.36-0.88)
\end{array}
$$

Highest genetic education (reference none)

$$
\begin{array}{ll}
\text { Undergraduate } & 0.87(0.61-1.24) \\
\begin{array}{l}
\text { During } \\
\text { specialist }
\end{array} & 1.10(0.56-2.18) \\
\text { training } & \\
\text { CME } & 0.73(0.45-1.19)
\end{array}
$$

Value of genetic education (reference useless)

$$
\begin{array}{lc}
\text { Useful } & 1.48(1.05-2.09) \\
\text { undergraduate } & \\
\text { Useful } & 3.77(0.44-31.96) \\
\text { specialist } & \\
\text { training } & \\
\text { Useful CME } & 0.73(0.14-3.77) \\
\text { Country (reference UK) } & \\
\text { France } & 4.01(1.82-8.80) \\
\text { Germany } & 23.97(11.29-50.87) \\
\text { Netherlands } & 7.76(3.63-16.62) \\
\text { Sweden } & 5.58(2.59-12.03) \\
\text { Gender (reference male) } \\
\text { Female } & 0.58(0.43-0.77) \\
\text { Age (reference >50) } & \\
\leq 50 & 1.06(0.82-1.37)
\end{array}
$$

$\begin{array}{ll}\text { France } & 2.95(1.85-4.70) \\ \text { Germany } & 1.64(1.02-2.63) \\ \text { Netherlands } & 1.31(0.81-2.13) \\ \text { Sweden } & 1.38(0.85-2.21)\end{array}$


Table 4 (continued)

\begin{tabular}{|c|c|c|}
\hline Task & Variable & $\begin{array}{l}\text { Odds ratio for doing } \\
\text { oneself }(95 \% \mathrm{CI})\end{array}$ \\
\hline
\end{tabular}

Informing Mr Smith of the implications if a mutation were to be found

Ordering the genetic test

$$
\begin{array}{lr}
\text { Years in practice }(\text { reference }>20) \\
11-20 & 1.02(0.78-1.35) \\
\leq 10 & 0.65(0.43-0.98)
\end{array}
$$

Highest genetic education (reference none)

$\begin{array}{ll}\text { Undergraduate } & 0.99(0.71-1.40) \\ \text { During } & 1.53(0.81-2.88) \\ \quad \text { specialist } & \\ \text { training } & \\ \text { CME } & 1.09(0.70-1.70)\end{array}$

Value of genetic education (reference useless)

$$
\begin{array}{ll}
\text { Useful } & 1.27(0.93-1.74) \\
\text { undergraduate } & \\
\text { Useful } & 0.68(0.17-2.69) \\
\text { specialist } & \\
\text { training } & \\
\text { Useful CME } & 0.61(0.14-2.66)
\end{array}
$$

Country (reference UK)

$$
\begin{array}{ll}
\text { France } & 4.46(1.83-10.89) \\
\text { Germany } & 8.51(3.58-20.20) \\
\text { Netherlands } & 3.42(1.39-8.42) \\
\text { Sweden } & 4.64(1.92-11.21) \\
\text { Gender (reference } & \text { male) } \\
\text { Female } & 0.52(0.36-0.76) \\
\text { Age (reference }>50) & \\
\leq 50 & 0.85(0.61-1.18) \\
\text { Years in practice }(\text { reference }>20) \\
11-20 & 0.84(0.60-1.18) \\
\leq 10 & 0.56(0.33-0.96)
\end{array}
$$

\begin{tabular}{|c|c|c|}
\hline Task & Variable & $\begin{array}{l}\text { Odds ratio for doing } \\
\text { oneself }(95 \% \mathrm{CI})\end{array}$ \\
\hline & \multicolumn{2}{|c|}{$\begin{array}{l}\text { Highest genetic education (reference } \\
\text { none) }\end{array}$} \\
\hline & Undergraduate & $1.24(0.80-1.90)$ \\
\hline & $\begin{array}{l}\text { During } \\
\text { specialist } \\
\text { training }\end{array}$ & $0.92(0.38-20.23)$ \\
\hline & CME & $1.15(0.66-2.02)$ \\
\hline
\end{tabular}

Highest genetic education (reference none)

$\begin{array}{ll}\text { Undergraduate } & 1.32(0.84-2.07) \\ \text { During } & 1.49(0.66-3.40) \\ \text { specialist } & \\ \text { training } & \\ \text { CME } & 1.18(0.66-2.13)\end{array}$

Value of genetic education (reference useless)

$$
\begin{array}{ll}
\text { Useful } & 1.36(0.92-2.01) \\
\text { undergraduate } & \\
\text { Useful } & 1.77(0.20-15.52) \\
\text { specialist } & \\
\text { training } & \\
\text { Useful CME } & 0.23(0.05-1.04)
\end{array}
$$

Country (reference UK)

$$
\begin{array}{ll}
\text { France } & 2.16(1.11-4.20) \\
\text { Germany } & 3.33(1.76-6.33) \\
\text { Netherlands } & 1.76(0.90-3.46) \\
\text { Sweden } & 2.25(1.17-4.33)
\end{array}
$$

Gender (reference male)

$$
\text { Female } \quad 0.62(0.43-0.88)
$$

Age (reference $>50$ )

$$
\leq 50 \quad 0.85(0.62-1.17)
$$

Years in practice (reference $>20$ )

$$
\begin{array}{ll}
11-20 & 0.94(0.67-1.32) \\
\leq 10 & 0.72(0.44-1.19)
\end{array}
$$

Table 4 (continued)

Value of genetic education (reference useless)

$$
\begin{array}{lc}
\begin{array}{l}
\text { Useful } \\
\text { undergraduate }
\end{array} & 1.29(0.88-1.87) \\
\text { Useful } & 0.35(0.08-1.65) \\
\text { specialist } & \\
\text { training } & \\
\text { Useful CME } & 0.55(0.11-2.89) \\
\text { Country (reference } & \text { UK) } \\
\text { France } & 5.45(1.87-15.87) \\
\text { Germany } & 10.24(3.62-28.95) \\
\text { Netherlands } & 3.55(1.20-10.56) \\
\text { Sweden } & 4.12(1.41-12.08)
\end{array}
$$

Explaining the test result

Gender (reference male)

Female $\quad 0.36(0.22-0.57)$

Age (reference $>50$ )

$$
\leq 50 \quad 0.73(0.51-1.06)
$$

Years in practice (reference $>20$ )

$$
\begin{array}{ll}
11-20 & 0.86(0.58-1.28) \\
\leq 10 & 0.68(0.38-1.22)
\end{array}
$$

Highest genetic education (reference none)

$\begin{array}{ll}\text { Undergraduate } & 1.47(0.88-2.45) \\ \text { During } & 0.80(0.26-2.46) \\ \begin{array}{l}\text { specialist } \\ \text { training }\end{array} & \\ \text { CME } & 0.90(0.44-1.83)\end{array}$

Value of genetic education (reference useless)

$$
\begin{array}{ll}
\text { Useful } & 1.05(0.69-1.60) \\
\text { undergraduate } & \\
\text { Useful } & \text { NA } \\
\text { specialist } & \\
\text { training } & \\
\text { Useful CME } & 0.25(0.05-1.35) \\
\text { Country (reference UK) }
\end{array}
$$

Explaining the implications of the test result for the children

$\begin{array}{lr}\text { France } & 10.58(2.48-45.19) \\ \text { Germany } & 16.52(3.94-69.25) \\ \text { Netherlands } & 9.05(2.12-38.70) \\ \text { Sweden } & 7.21(1.67-31.09)\end{array}$

Gender (reference male)

Female

Age (reference $>50$ )

$$
\leq 50 \quad 0.81(0.56-1.19)
$$

Years in practice (reference $>20$ )

$$
\begin{array}{ll}
11-20 & 0.87(0.58-1.31) \\
\leq 10 & 0.82(0.46-1.44)
\end{array}
$$

Highest genetic education (reference none)

Undergraduate $1.05(0.64-1.73)$ 
Table 4 (continued)

\begin{tabular}{|c|c|c|}
\hline Task & Variable & $\begin{array}{l}\text { Odds ratio for doing } \\
\text { oneself }(95 \% \mathrm{CI})\end{array}$ \\
\hline & $\begin{array}{l}\text { During } \\
\text { specialist } \\
\text { training }\end{array}$ & $0.88(0.32-2.43)$ \\
\hline & $\mathrm{CME}$ & $0.84(0.42-1.66)$ \\
\hline & \multicolumn{2}{|c|}{$\begin{array}{l}\text { Value of genetic education (reference } \\
\text { useless) }\end{array}$} \\
\hline & $\begin{array}{l}\text { Useful } \\
\text { undergraduate }\end{array}$ & $1.30(0.83-2.06)$ \\
\hline & $\begin{array}{l}\text { Useful } \\
\text { specialist } \\
\text { training }\end{array}$ & $0.98(0.11-9.14)$ \\
\hline & Useful CME & $0.69(0.08-5.98)$ \\
\hline
\end{tabular}

rather than psychosocial information (Roter et al. 2002). Also, the self-reporting nature of this study may be affected by the tendency of female physicians to under-rate their own competence (Nomura et al. 2010).

Table 5 Multivariate analysis

\begin{tabular}{|c|c|c|c|}
\hline Task & $\begin{array}{l}\text { Factors predictive } \\
\text { of doing it oneself }\end{array}$ & Wald score & $P$ \\
\hline $\begin{array}{l}\text { Taking a family } \\
\text { history }\end{array}$ & Country & 193.05 & $<0.005$ \\
\hline \multirow{3}{*}{$\begin{array}{l}\text { Explaining the inheritance } \\
\text { pattern }\end{array}$} & Country & 25.68 & $<0.005$ \\
\hline & Age & 7.12 & 0.008 \\
\hline & $\begin{array}{l}\text { Quality of } \\
\text { undergraduate } \\
\text { education }\end{array}$ & 12.60 & $<0.005$ \\
\hline \multirow{2}{*}{$\begin{array}{l}\text { Explaining the risk to } \\
\text { Mr Smith's children }\end{array}$} & Country & 24.04 & $<0.005$ \\
\hline & $\begin{array}{l}\text { Quality of } \\
\text { undergraduate } \\
\text { education }\end{array}$ & 7.12 & 0.008 \\
\hline \multirow[t]{3}{*}{$\begin{array}{l}\text { Giving information about } \\
\text { available gene tests }\end{array}$} & $\begin{array}{l}\text { Quality of } \\
\text { undergraduate } \\
\text { education }\end{array}$ & 6.29 & 0.012 \\
\hline & Gender & 4.59 & 0.032 \\
\hline & Age & 6.40 & 0.011 \\
\hline \multirow{2}{*}{$\begin{array}{l}\text { Informing Mr Smith of the } \\
\text { implications if no mutation } \\
\text { were to be found }\end{array}$} & Country & 93.09 & $<0.005$ \\
\hline & Gender & 6.16 & 0.013 \\
\hline \multirow{2}{*}{$\begin{array}{l}\text { Informing Mr Smith of the } \\
\text { implications if a mutation } \\
\text { were to be found }\end{array}$} & Country & 31.02 & $<0.005$ \\
\hline & Gender & 9.51 & 0.002 \\
\hline \multirow[t]{2}{*}{ Ordering the genetic test } & Country & 15.07 & 0.005 \\
\hline & Gender & 7.22 & 0.007 \\
\hline \multirow[t]{2}{*}{ Explaining the test result } & Country & 29.24 & $<0.005$ \\
\hline & Gender & 15.05 & $<0.005$ \\
\hline \multirow{2}{*}{$\begin{array}{l}\text { Explaining the implications } \\
\text { of the test result for the } \\
\text { children }\end{array}$} & Country & 19.51 & 0.001 \\
\hline & Gender & 7.93 & 0.005 \\
\hline
\end{tabular}

This is to our knowledge the first study in Europe of primary care providers' attitudes to genetic management and how they relate to genetic education. Although the response rate was not high, this is a common problem for postal surveys and all appropriate methods were used to increase the response rates. Databases from which samples were taken varied slightly between countries, but represented the only available national sources with doctors' addresses and specialties. We recognise that we have studied self-reported rather than actual behaviour but analysis of actual behaviour would have been impossible to be organised practically and self-reporting can be considered as a reliable proxy measure. Although the scenario used related only to one condition, sudden death from hypertrophic cardiomyopathy was selected as a scenario diagnosis specifically because it was unlikely to have featured in traditional Mendelian genetics teaching. The importance of genetics in its aetiology is, however, well recognised. We therefore suggest that it is likely to be a good model for common complex disorders with genetic aetiology encountered by primary care providers.

We have previously demonstrated that genetic care by non-geneticists is patchy and often poorly documented (Lane et al. 1997; Williamson et al. 1997; Williamson et al. 1996a, b). This is supported by qualitative research which found highly variable levels of information around referral and testing for Factor V Leiden (Saukko et al. 2007) and multiple potential barriers to effective communication amongst GPs providing antenatal counselling (Nagle et al. 2008). Our work shows clearly that, apart from family history taking, many European GPs do not consider that "genetic" care should form part of their practice.

\section{Conclusions}

It is clear that given the significant effect of country of practice, independent of all other factors, on practitioner behaviour, recommendations on genetic education at all levels will have to be sensitive to country-specific issues. Educational structures and content will require tailoring to local priorities and learning conventions. Any standards of care for non-genetic specialists providing some aspects of genetic care will need to be appropriately contextualised into the local system of health care and health education and it is unlikely that a pan-European "one size fits all" policy will be immediately workable or acceptable.

Acknowledgements Thanks to Karina Bertmaring, Daniel Cottam and Christine Waterman who provided invaluable administrative and data management support. The study was funded by European Community FP5 grant QLG4-CT-2001-30216. 


\section{Conflicts of interest None.}

Authors' contributions RH, HH, IN, JS, LPtK, UK and CJ-R conceived the study. All authors were involved in questionnaire construction, statistical analysis and drafting of the manuscript.

Open Access This article is distributed under the terms of the Creative Commons Attribution Noncommercial License which permits any noncommercial use, distribution, and reproduction in any medium, provided the original author(s) and source are credited.

\section{References}

Baars M, De Smit D, Langendam M, Ader H, ten Kate L (2003) Comparison of activities and attitudes of general practitioners concerning genetic counseling over a 10-year time-span. Patient Educ Couns 50(2):145-149

Barrison A, Smith C, Oviedo J, Heeren T, Schroy PR (2003) Colorectal cancer screening and familial risk: a survey of internal medicine residents' knowledge and practice patterns. Am J Gastroenterol 98(6):1410-1416

Batra S, Valdimarsdottir H, McGovern M, Itzkowitz S, Brown K (2002) Awareness of genetic testing for colorectal cancer predisposition among specialists in gastroenterology. Am J Gastroenterol 97(3):729-733

Calefato J-M, Nippert I, Harris H, Kristoffersson U, Schmidtke J, Ten Kate L et al (2008) Assessing educational priorities in genetics for GP's and specialists in 5 countries: factor structure of the genetic educational priorities (Gen-EP) scale. Genet Med 10:99-106

Calzone K, Jenkins J, Masny A (2002) Core competencies in cancer genetics for advanced practice oncology nurses. Oncol Nurs Forum 29(9): 1327-1333

Challen K, Harris H, Julian-Reynier C, Ten Kate L, Kristoffersson U, Nippert I et al (2005) Genetic education and non-genetic health professionals: educational providers and curricula in Europe. Genet Med 7:302-310

Challen K, Harris H, Benjamin CM, Harris R (2006) Genetics teaching for non-geneticist health care professionals in the UK. Community Genet 9:251-259

Core Competency Working Group of the National Coalition for Health Professional Education in Genetics (2001) Recommendations of core competencies in genetics essential for all health professionals. Genet Med 3(2):155-159

Department of Health (2003) Our inheritance, our future (No. Cm5791-II). Department of Health, London

Department of Health (2005) National service framework for coronary heart disease. Department of Health, London

Emery J, Watson E, Rose P, Andermann A (1999) A systematic review of the literature exploring the role of primary care in genetic services. Fam Pract 16(4):426-445

Greendale K, Pyeritz R (2001) Empowering primary care health professionals in medical genetics: how soon? How fast? How far? Am J Med Genet 106(3):223-232

Guttmacher A, Collins F (2002) Genomic medicine: a primer. NEJM 347(19):1512-1520

Harris R, Harris H (1995) Primary care for patients at genetic risk. BMJ 311:579-580

Harris R, Lane B, Harris H, Williamson P, Dodge J, Modell B et al (1999) National confidential enquiry into counselling for genetic disorders by non-geneticists: general recommendations and specific standards for improving care. Br J Obs Gynae 106:658-663
Henriksson K, Kristoffersson U (2006) Education in medical genetics for non-genetic health care providers in Sweden. Community Genet 9:240-245

Hosmer DW, Lemeshow S (2000) Applied logistic regression, 2nd edn. Wiley-Interscience, New York

Julian-Reynier C, Arnaud S (2006) France: genetics education for non-genetics health care providers. Community Genet 9:227-234

Julian-Reynier C, Nippert I, Calefato J-M, Harris H, Kristoffersson U, Schmidtke J et al (2008) Genetics in clinical practice: general practitioners' educational priorities in European countries. Genet Med 10:107-113

Kirk M, McDonald K, Longley M, Anstey S, Anionwu E, Benjamin C et al (2003) Fit for practice in the genetics era: a competence based education framework for nurses, midwives and health visitors. University of Glamorgan, Pontypridd

Lane B, Williamson P, Dodge J, Harris H, Super M, Harris R (1997) Confidential inquiry into families with two siblings with cystic fibrosis. Arch Dis Child 77:501-503

McNally E, Cambon-Thomsen A, on behalf of EC expert group (2004) 25 Recommendations on the ethical, legal and social implications of genetic testing. European Commission, Brussels

Nagle C, Lewis S, Meiser B, Gunn J, Halliday J, Bell R (2008). Exploring general practitioners' experience of informing women about prenatal screening tests for foetal abnormalities: a qualitative focus group study. BMC Health Serv Res 8(114)

Nomura K, Yano E, Fukui T (2010) Gender differences in clinical confidence: a nationwide survey of resident physicians in Japan. Acad Med 85:647-653

Plass AMC, Baars MJ, Beemer FA, Kate LPT (2006) Genetics education for non-genetic health care professionals in the Netherlands. Community Genet 9:246-250

Roter DL, Hall JA, Aoki Y (2002) Physician gender effects in medical communication: a meta-analytic review. JAMA 288:756-764

Saukko PM, Ellard S, Richards SH, Shepherd MH, Campbell JL (2007) Patients' understanding of genetic susceptibility testing in mainstream medicine: qualitative study on thrombophilia. BMC Health Serv Res 7(82)

Schmidtke J, Paul Y, Nippert I (2006) Education in medical genetics for physicians: Germany. Community Genet 9:235-239

Schroy PR, Barrison A, Ling B, Wilson S, Geller A (2002) Family history and colorectal cancer screening: a survey of physician knowledge and practice patterns. Am J Gastroenterol 97(4):1031-1036

Taylor M (2003) A survey of chairpersons of departments of medicine about the current and future roles of clinical genetics in internal medicine. Genet Med 5(4):328-331

Tyzack K, Wallace E (2003) Down syndrome screening: what do health professionals know? Aust N Z J Obstet Gynaecol 43 (3):217-221

van Langen I, Birnie E, Leschot N, Bonsel G, Wilde A (2003) Genetic knowledge and counselling skills of Dutch cardiologists: sufficient for the genomics era? Eur Heart J 24(6):560-566

Williamson P, HArris R, Church S, Fiddler M, Rhind J (1996a) Prenatal genetic services for down's syndrome: access and provision in 1990-1991. Br J Obstet Gynaecol 103:676-683

Williamson P, Ponder B, Church S, Fiddler M, Harris R (1996b) The genetic aspects of medullary thyroid carcinoma: recognition and management. J R Coll Physicians Lond 30:443-447

Williamson P, Alberman E, Rodeck C, Fiddler M, Church S, Harris R (1997) Antecedent circumstances surrounding neural tube defect births in 1990-1991. Br J Obstet Gynaecol 104:51-56

World Alliance of Organizations for the Prevention of Birth Defects (2004) Prevention of birth defects: a task for a world alliance. Retrieved 11th May 2004

Yong M, Zhou X, Lee S (2003) The importance of paternal family history in hereditary breast cancer is underappreciated by health care professionals. Oncology 64(3):220-226 